\title{
RESEARCH PAPER RP889
}

Part of Journal of Research of the National Bureau of Standards, Volume 16, June 1936

\section{CALCULATION OF THE CONCENTRATION AND DISSOCIA- TION CONSTANT OF EACH ACID GROUP IN A MIXTURE FROM THE $\mathrm{pH}$ TITRATION CURVE OF THE MIXTURE}

\author{
By J. O. Burton and S. F. Acree
}

\section{ABSTRACT}

Experimental methods and equations are given for calculating the concentration and dissociation constant of each acid or base in a mixture by use of its complex $\mathrm{pH}$ titration curve. The principle is illustrated by means of experimental data for malonic acid.

This problem is important as an aid in determining the nature of the acids and bases in plant and animal fibers, fruits, culture media, pulp, paper, and products derived from them.

\section{CONTENTS}

I. Introduction

II. Application of equations

1. Dissociation constant of first acid _.

2. Concentrations and dissociation constants of the other acids .... 528

III. References_............ 528

\section{INTRODUCTION}

The plot of the $\mathrm{pH}$ values against the mole fractions of neutralization of a given concentration of any acid or base at a given temperature is called its $\mathrm{pH}$ titration curve, a mixture of acids or bases giving a corresponding complex $\mathrm{pH}$ titration curve. Both liquids and solids may give such curves.

In earlier articles [1] ${ }^{1}$ mathematical equations and graphical methods were described by the use of which a complex $\mathrm{pH}$ titration curve for a mixture of acids or bases can be resolved into the individual $\mathrm{pH}$ curve for each acid or base. In the same way the individual $\mathrm{pH}$ curves can be integrated into the total or complex $\mathrm{pH}$ curve for any known mixture of acids or bases within experimental errors.

General equations for calculating the values of the two dissociation constants, $K_{1}$ and $K_{2}$, from the pH titration curves for dibasic acids were then developed [2], and when applied to malonic acid gave

$$
K_{1}=2.06 \times 10^{-3} \text { and } K_{2}=2.94 \times 10^{-6} \text {. }
$$

The purpose of this article is to present an extension of the use of these equations for the calculation of the concentration and the dissociation constant of each acid or base in a mixture, and to illustrate the principle with data for malonic acid. This problem is important 
in determining the nature of the acids and bases in plant and animal fibers, fruits, culture media, pulp, paper, and products derived from them.

Whether the solution contains only one mono- or $n$-basic acid, $\mathrm{H}_{n} \mathrm{An}$, or $n$ different monobasic acids, or a mixture of mono- and polybasic acids, we may call the total equivalent concentration of acid $\left[\mathrm{H}_{n} \mathrm{An}\right]$ and let $a$ represent the fraction of $\left[\mathrm{H}_{n} \mathrm{An}\right]$ which corresponds to the strongest acid group. If the acid is a single polybasic one, the salts formed in sequence on addition of $\mathrm{NaOH}$ are $\mathrm{NaH}_{n-1} \mathrm{An}$, $\mathrm{Na}_{2} \mathrm{H}_{n-2} \mathrm{An}$, etc., whereas a mixture of monobasic acids in any proportions forms, in succession, the salts represented by NaAn, $\mathrm{NaAn}, \mathrm{NaAn}$ ', etc. A logical application of the law of mass action leads to no confusion in the calculation of the value of $a$ for each successively weaker acid in any of these mixtures, and hence we may point out the general principles by discussing the particular application of the law to malonic acid, for which the data are available [2].

\section{APPLICATION OF EQUATIONS}

Equation 1 gives the values of the primary dissociation constant, $K_{1}$, of a polybasic acid when $\left[\mathrm{H}_{n} \mathrm{An}\right]$ is the equivalent concentration of total acid, determined by titration with standard alkali, $a$ is the fraction corresponding to the strongest or primary acid group, $\left[\mathrm{H}^{+}\right]$is the hydrogen-ion concentration, $\alpha_{1}$ is the degree of ionization of the primary salt, and $\left[\mathrm{NaH}_{n-1} \mathrm{An}\right],\left[\mathrm{Na}_{2} \mathrm{H}_{n-2} \mathrm{An}\right]$, etc., are the molar concentrations of the salts. As no assumption of the basicity of the acid is involved this equation applies also to the strongest acid in a mixture.

$$
K_{1}=\frac{\left[\mathrm{H}^{+}\right]\left(\alpha_{1}\left[\mathrm{NaH}_{n-1} \mathrm{An}\right]+\left[\mathrm{H}^{+}\right]\right)}{a\left[\mathrm{H}_{n} \mathrm{An}\right]-\left[\mathrm{NaH}_{n-1} \mathrm{An}\right]-\left[\mathrm{Na}_{2} \mathrm{H}_{n-2} \mathrm{An}\right]-\cdots\left[\mathrm{H}^{+}\right]} .
$$

The parenthesis does not represent activities and is used here for simplicity. These symbols can be replaced by activities [2] to secure the thermodynamic-activity constant instead of the classical dissociation constant, $K_{1}$. Since the values of $a$ are obtained from a series of simultaneous equations discussed below and the ionic strength remains nearly constant, thus giving an approximately constant value [2] for the activity-coefficient product, $f_{\mathrm{H}} f_{\mathrm{H}_{n-1}} \mathrm{An}$, either the classical or the thermodynamic method may be employed. The former is used here because of its simplicity and direct adaptability to a simple analytical treatment of mixed acids.

Let $u$ represent the numerator of equation 1 at any given $\mathrm{pH}$ or $\left[\mathrm{H}^{+}\right]$and $v$ all the denominator except $a\left[\mathrm{H}_{n} \mathrm{An}\right]$. No values of $\left[\mathrm{H}^{+}\right]$need be considered which cause formation of secondary salt, and calculations are thereby simplified. $\left[\mathrm{NaH}_{n-1} \mathrm{An}\right]$ is then equivalent to the alkali added to give each $\left[\mathrm{H}^{+}\right]$value, correction being made for the dilution of the acid solution by the alkali.

We then have a series of siluultaneous equations in $a$ and $K_{1}$ for all values of $\left[\mathrm{H}^{+}\right]$, which we may write

$$
\mathrm{K}_{1}=\frac{u}{a\left[\mathrm{H}_{n} \mathrm{An}\right]-v}=\frac{u^{\prime}}{a\left[\mathrm{H}_{n} \mathrm{An}\right]^{\prime}-v^{\prime}} .
$$

When solved for $a$ we get

$$
a=\frac{u^{\prime} v-u v^{\prime}}{u^{\prime}\left[\mathrm{H}_{n} \mathrm{An}\right]-u\left[\mathrm{H}_{n} \mathrm{An}\right]^{\prime}} .
$$


This equation is simplified when $\left[\mathrm{H}_{n} \mathrm{An}\right]$ is kept constant experimentally by adding different mole fractions of alkali to fixed volumes of acid and diluting with pure water to a chosen value of $\left[\mathrm{H}_{n} \mathrm{An}\right]$, instead of changing it to $\left[\mathrm{H}_{n} \mathrm{An}\right]^{\prime}$ by addition of successive portions of alkali to one volume of acid to change the $\left[\mathrm{H}^{+}\right]$values, as was done in the work [2] on malonic acid, given in table 1. Smaller deviations are found for $a$ by adopting mathematical artifices [3] such as comparison of equation 2 for any one value of $\left[\mathrm{H}^{+}\right]$against the summation equations for all values of $\left[\mathrm{H}^{+}\right]$. When two simultaneous equations of the series (2) involve values of $\left[\mathrm{H}^{+}\right]$which are close together, small experimental errors in $u, u^{\prime}, v, v^{\prime}$, etc., may produce large percentage deviations in the numerator and denominator of equation 3 and hence in $a$. The smallest errors in $a$ are, therefore, obtained when wide differences in $\left[\mathrm{H}^{+}\right]$are used in equation 3 . The summation method was used with the values of $\left[\mathrm{H}^{+}\right]$and $\mathrm{NaOH}$ found in the earlier article [2] on malonic acid $(0.1 M)$, and some of the results for $a$ are given in table 1 . It is seen that the average value of $a, 0.484$, is very close to the theoretical value, 0.5 , for the dibasic malonic acid. In other words, if $a$ for the strongest acid group is found by this method to be approximately 0.5 for an unknown pure acid, it is a dibasic acid; if $a$ is found to be approximately 0.33 or 0.25 , it is a tribasic or tetrabasic acid, respectively. This emf method for determining the basicity of any acid supplements data on the number of its salts and esters and on its structural formula.

TABLE 1.-Calculated values of $a$ and $K_{1}$ for $0.1 \mathrm{M}$ malonic acid 1

\begin{tabular}{|c|c|c|c|c|c|c|c|c|}
\hline $\begin{array}{l}\text { Equiva- } \\
\text { lents of } \\
\mathrm{NaOH}\end{array}$ & $\begin{array}{c}\text { Equivalent } \\
\text { concentra-s } \\
\text { tion Na salt }\end{array}$ & $\begin{array}{c}a\left[\mathrm{H}_{n} \mathrm{An}\right]+ \\
\text { Na salt } \\
\text { (equivalents) }\end{array}$ & {$\left[\mathrm{H}^{+}\right] \times 10^{3}$} & $\alpha_{1}$ & $a$ & $\begin{array}{c}K_{1} \times 10^{3} \\
\text { from equa- } \\
\text { tion } 2\end{array}$ & \multicolumn{2}{|c|}{$\begin{array}{c}K_{1} \times 10^{3} \text { from equa- } \\
\text { tion } 1\end{array}$} \\
\hline $\begin{array}{c}0.1294 \\
.2059 \\
.3667 \\
.5279 \\
.6891 \\
.7697 \\
.8100\end{array}$ & $\begin{array}{r}0.011461 \\
.017090 \\
.026873 \\
.034624 \\
.040902 \\
.043614 \\
.044880\end{array}$ & $\begin{array}{r}0.088621 \\
.083029 \\
.073314 \\
.065617 \\
.059382 \\
.056690 \\
.055432\end{array}$ & $\begin{array}{c}7.9433 \\
6.0255 \\
3.3113 \\
1.8621 \\
.9550 \\
.64565 \\
.51286\end{array}$ & $\begin{array}{l}0.927 \\
.911 \\
.891 \\
.878 \\
.868 \\
.865 \\
.863\end{array}$ & $\begin{array}{l}0.491 \\
.476 \\
.509 \\
.467 \\
.464 \\
.490 \\
.494\end{array}$ & $\begin{array}{l}2.18 \\
2.33 \\
2.03 \\
2.42 \\
2.62 \\
2.19 \\
2.15\end{array}$ & $\begin{array}{l}2.13 \\
2.17 \\
2.09 \\
2.06 \\
1.99 \\
1.99 \\
2.01\end{array}$ & $\begin{array}{l}(2.06) \\
(2.06) \\
(2.04) \\
(1.99) \\
(2.00) \\
(1.97) \\
(2.00)\end{array}$ \\
\hline A verage. . & & & & & $\ldots . .484$ & 2. $27 \pm 0.16$ & $2.06=$ & $6(2.02) \pm 0.03$ \\
\hline
\end{tabular}

1 The values in parentheses are those obtained after the emf has been corrected for liquid junction poten. tial and interionic attraction between ions of unlike sign [2]. The values of $a$ in column 6 vary, owing primarily to the fact that they were obtained without the use of activity coefficients. However, the conclusions as to the significance of the magnitude of $a$ remain unchanged. Since the important point of this paper is the determination of the type of an acid in an unknown, the liquid-junction potentials are not considered as was done [2] earlier. Accurate corrections with an unknown are not possible, but they are generally very small when saturated potassium chloride is employed for the solution of the reference calomel electrode.

Equations 2 and 3 are valuable in estimating the concentration of a solution of a single, weak monobasic acid HAn instead of relying upon a colorimetric, electrometric, or differential electrometric end point involving rapidly changing $\mathrm{pH}$ values. In this case $a=1$ and the value of $\left[\mathrm{H}_{n} \mathrm{An}\right]$, that is, [HAn] is sought. If [HAn] is kept constant and equal to $\left(u^{\prime} v-u v^{\prime}\right) /\left(u^{\prime}-u\right)$ from equation 3 , its value can be determined by measuring out the standard solution of $\mathrm{NaOH}$ by weight, and using precision methods for evaluating $\left[\mathrm{H}^{+}\right]$in a linear accurate $\mathrm{pH}$ titration curve without even reaching the end point. Even when $[\mathrm{HAn}]^{\prime}$ is the known $x$ fraction of $[\mathrm{HAn}]$ at any $\left[\mathrm{H}^{+}\right]$, from equation 3 [HAn] is equal to $\left(u^{\prime} v-u v^{\prime}\right) /\left(u^{\prime}-x u\right)$, and can be found accurately by taking its average for several values of $\left[\mathrm{H}^{+}\right]$. Then $K_{1}$ can be calculated for the weak acid from equation 1 . 


\section{DISSOCIATION CONSTANT OF FIRST ACID}

The various simultaneous equations 2 used to determine $a$ can also be solved for $K_{1}$ in this treatment. We may, however, simply substitute the value found for $a$ at the given $\left[\mathrm{H}^{+}\right]$, or preferably the average value of $a$, in the corresponding equation 2 and solve for $K_{1}$. The results of the first method are given in column 7 of table 1 and are to be compared with values of $K_{1}$ obtained from equation 1 . We note that, in general, $K_{1}$ from equation 2 rises as $a$ decreases. The average value of $K_{1}$ obtained from equation 2 is slightly higher than that from equation 1 , because the average $a$ for the $\left[\mathrm{H}^{+}\right]$values chosen is slightly less than the theoretical value 0.5 . When 0.5 is used for $a$ in equation 2 , the values of $K_{1}$ obtained are very close to those from equation 1. If the value $K_{1}=2.06 \times 10^{-3}$ is employed, the $a$ values are, respectively, $0.513,0.520,0.505,0.500,0.499,0.501$, and 0.498 , and their average is 0.505 .

\section{CONCENTRATIONS AND DISSOCIATION CONSTANTS OF THE OTHER ACIDS}

It is clear, then, that within the experimental errors both the mole fraction and the dissociation constant of the strongest acid group in a mixture can be calculated by this method. The equations can then be applied as follows to successive portions of the $\mathrm{pH}$ curve to calculate in turn the concentration and dissociation constant of each successively weaker acid. The values of $K_{1}$ obtained for successive additions of standard alkali show a sharp increase upon formation of the second salt, whose concentration can then be calculated as previously described [2]. By use of the values of $\left[\mathrm{H}^{+}\right]$and the concentrations of secondary salt found for the overlapping $\mathrm{pH}$ titration curves of the first and second acids, and for that portion of the $\mathrm{pH}$ curve for the second acid not overlapping those for the first and third acids, the magnitude of $K_{2}$ and $a$ for the second acid can be calculated as above for the first acid. These values of $K_{2}$ and $a$ remain constant until formation of the salt of the third acid, for which $K_{3}$ and $a$ can then be calculated.

It should also be pointed out that in those portions of the $\mathrm{pH}$ curve involving overlapping of the individual $\mathrm{pH}$ curves for two acids undergoing simultaneous neutralization, the denominator of equation 1 is related to the amount of added alkali in two different ways, depending on whether a mixture of monobasic acids or a polybasic acid is concerned. This method is therefore useful as a means of analysis of mixed acids, whether mono- or polybasic.

The authors wish to express their appreciation to Dr. Walter J. Hamer for helpful criticism in the preparation of this paper.

\section{REFERENCES}

[1] Mellon, Avery, Slagle, and Acree, J. Infect. Diseases 29, 1, 75 (1921).

[2] J. O. Burton, W. J. Hamer, and S. F. Acree, J. Research NBS 16, 575 (1936) RP895.

[3] E. K. Marshall and S. F. Acree, J. Phys. Chem. 19, 589 (1915); W. A. Taylor and S. F. Acree, J. Phys. Chem. 20, 365 (1916):

Washington, April 18, 1936. 